Franke, J./ Pfaff, D./ Elbert, R./ Gomm, M./ Hofmann, E. (2005):

Die Financial Chain im Supply Chain Management: Konzeptionelle Einordnung und Identifikation von Werttreibern.

In: Ferstel, O. K./ Sinz, E. J./ Eckert, S./ Isselhorst, T. (Hrsg.): Wirtschaftsinformatik 2005. eEconomy, eGovernment, eSociety. Heidelberg 2005, S. 567-584

Dr. Erik Hofmann

Kühne-Institut für Logistik (KLOG-HSG)

Universität St.Gallen

Dufourstrasse 40a

CH-9000 St.Gallen 


\title{
Die Financial Chain im Supply Chain Management: Konzeptionelle Einordnung und Identifikation von Werttreibern
}

\author{
Jochen Franke, Donovan Pfaff \\ Universität Frankfurt am Main \\ Ralf Elbert, Moritz Gomm \\ Technische Universität Darmstadt \\ Erik Hofmann \\ Universität St. Gallen
}

Zusammenfassung: Die Verflechtung von Industrie- und Handelsunternehmen mit Finanzdienstleistern wächst. Ebenfalls nimmt die Industrialisierung der Finanzwelt stetig zu. Das aus dem Supply Chain Management hervorgehende Financial Chain Management adressiert Fragestellungen der Finanzflüsse in und zwischen Industrie- und Handelsunternehmen sowie Finanzdienstleistern. Um zielgerichtet Verbesserungspotenziale in den Finanzflüssen zu realisieren, spielt die Identifizierung der maßgeblichen Werttreiber eine entscheidende Rolle. Basierend auf einer empirischen Studie unter den 1.000 umsatzstärksten Industrieund Handelsunternehmen in Deutschland werden exemplarisch für die Financial Chain Werttreiber aufgezeigt. Für die Teilprozesse Qualifikation und Rechnungsstellung zeigt sich, dass eine Reduktion der Prozessdauer den Unternehmenswert signifikant erhöhen kann.

Schlüsselworte: Financial Chain Management, Finanzflüsse, Werttreiber

\section{Einleitung}

Die prozessuale Verflechtung von Industrie- und Handelsunternehmen mit Finanzdienstleistern wächst. Dies zeigt sich unter anderem durch Initiativen der Banken zur Übernahme der Rechnungsstellung (e-bills der Deutschen Bank) oder des Cash Management (z.B. COCMS der Commerzbank) für Industrie- und Handelsunternehmen. Zudem nimmt die Industrialisierung der Finanzwelt stetig zu [KePf04]. Gerade in der Untersuchung von Vorgängen über die gesamte Wertschöpfungskette hinweg kommt in Wissenschaft und Praxis den 
finanzwirtschaftlichen Aspekten eine immer größere Bedeutung zu [Pfaf $+04 \mathrm{~b}$; Pfoh+03].

Die Untersuchung der Steuerung des Güterflusses im Rahmen des Supply Chain Managements stellt eine bedeutende Forschungsdisziplin innerhalb der Betriebswirtschaftslehre dar. Im Wettbewerb konkurrieren heute weniger einzelne

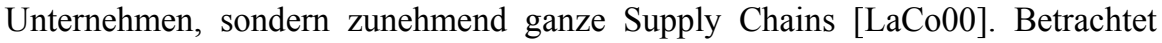
werden im Rahmen des Supply Chain Managements Fragestellungen der möglichst reibungslosen Beschaffung, Produktion und Distribution physischer Güter sowie der informationstechnischen und organisatorisch-rechtlichen Integration der an der gesamten Wertschöpfungskette beteiligten Unternehmen [Ball +00$]$.

Die Analyse und Steuerung der physischen Leistungserstellungsprozesse im Rahmen des Supply Chain Managements wird als kritische Größe für den Unternehmenserfolg gesehen [Lamb+98]. Der Gegenstrom zur physischen Reallokation von Gütern, der Fluss von Zahlungsmitteln innerhalb einer die Supply Chain begleitenden Financial Chain ist bislang nur in wenigen Arbeiten adressiert worden (beispielhaft [Kill02; Pfaf $+04 a$; Pfoh +03$]$ ). Insbesondere die Identifizierung von potenziellen Werttreibern in Prozessen spielt eine entscheidende Rolle, um zielgerichtet Wertsteigerungspotenziale $\mathrm{zu}$ quantifizieren. Das Working Capital Management und die damit verbundene Reduzierung von Außenständen innerhalb der Financial Chain sei hierfür als Beispiel angeführt. Hier setzen auch zahlreiche Finanzdienstleister mit entsprechenden Angeboten für ihre Firmenkunden an [Pfaf $+04 c$; EiJü03].

Der vorliegende Beitrag charakterisiert zunächst grundsätzlich die Interdependenzen von Finanzen und Logistik innerhalb des Supply Chain Managements und leitet daraus das Konzept des Financial Chain Managements ab. Dafür wird die generische Financial Chain als spezieller Teilbereich des Supply Chain Managements herausgegriffen und detailliert beschrieben. Anschließend werden exemplarisch Werttreiber für Teilprozesse der Financial Chain aus Verkäufersicht herausgegriffen und deren Wirkung im Rahmen einer empirischen Studie analysiert.

\section{Financial Supply Chain Management}

Während die materialwirtschaftlichen und insbesondere informationstechnischen Aspekte des Supply Chain Managements als Logistikkonzeption durch eine Vielzahl von Methoden und Instrumenten unterstützt werden, wird zunehmend nach Möglichkeiten gesucht, auch die finanzwirtschaftlichen Aspekte der Supply Chain zu berücksichtigen und zu optimieren. Diese, innerhalb und zwischen Unternehmen fließenden Finanzflüsse mit ihren Quellen und Senken, werden 
häufig als „Financial Supply Chain“ zusammengefasst [Pfaf +03$]$. Der folgende Abschnitt konkretisiert den Bereich des Financial Supply Chain Managements als Erweiterung des „klassischen“ Supply Chain Managements und zeigt Interdependenzen zwischen beiden auf. Die Financial Chain wird anschließend als Teilbereich des Financial Supply Chain Managements eingeordnet.

\subsection{Logistik und das klassische Supply Chain Management- Verständnis}

Nachdem die Integration der Material- und Informationsströme in der Forschung seit langem diskutiert und auch in der unternehmerischen Praxis umgesetzt wird, tritt die Integration sowie das Management finanzieller Mittel in den Vordergrund der Supply Chain Management-Diskussion [Pfoh+03].

Im Sinne einer Gesamtoptimierung der Prozesse ergeben sich neue Herausforderungen sowie Wert- und Leistungssteigerungspotenziale für die Akteure der Supply Chain und damit neue Geschäftsfelder für Banken, Finanzund Logistikdienstleister. Im Zentrum des Interesses steht dabei die Untersuchung des Finanzmittelflusses in der Supply Chain. Dieser Fluss ist das Bindeglied von logistischen und finanzwirtschaftlichen (Dienst-)Leistungen und konstitutives Element der Financial Supply Chain. Ziel ist es, durch das Management der Financial Supply Chain den Unternehmenswert der einzelnen Supply ChainAkteure und damit die Wettbewerbsfähigkeit der Supply Chain als Ganzes zu steigern.

Im Folgenden werden zunächst die Begriffe Logistik und Supply Chain Management abgegrenzt. Zur Definition von Supply Chain Management und der Abgrenzung zur Logistik siehe auch [LaHa04].

\section{Logistik}

Im Rahmen dieses Beitrages wird Logistik entsprechend der flussorientierten Definition des Council of Logistics Management (CLM) als „Prozess der Planung, Realisierung und Kontrolle des effizienten, kosteneffektiven Fließens und Lagerns von Rohstoffen, Halbfabrikaten und Fertigfabrikaten und der damit zusammenhängenden Information vom Liefer- zum Empfangspunkt entsprechend den Anforderungen des Kunden" verstanden [CLM04]. Diese Definition verweist auf den Logistikprozess, der in einem System zur raum-zeitlichen Transformation von Informationen und Gütern, dem so genannten Logistiksystem, abläuft. In einer funktionalen Betrachtung von Logistiksystemen ergeben sich die verrichtungsspezifischen Subsysteme Auftragsabwicklungssystem, Lagerhaltungssystem, Lagerhaussystem, Verpackungssystem und Transportsystem [Pfoh04].

Eine weitere Möglichkeit ist eine Differenzierung nach den Phasen des Güterflusses eines Unternehmens. Dadurch erhält man die phasenspezifischen 
Subsysteme der Logistik. Dies sind, bezogen auf die Unternehmenslogistik, die Beschaffungs-, Produktions-, Distributions-, Entsorgungs- und die ErsatzteilLogistik [Pfoh04].

\section{Klassisches Supply Chain Management-Verständnis}

Der Begriff Supply Chain ist in der Literatur unterschiedlich definiert (vgl. [Coop+97; Harr95]. Die Supply Chain kann als Bindeglied zwischen Herstellern, deren Zulieferern, dem Groß- und Einzelhandel als Absatzmittler, zwischengeschalteten Logistikunternehmen sowie dem Endabnehmer beschrieben werden. Das Supply Chain Management basiert auf dem Grundgedanken der Flussorientierung [Pfoh04], d.h. der Betrachtung von Güter- und Informationsströmen durch das Unternehmen. Dabei werden über Güter/Waren und logistische Informationen als Objekte hinaus auch akquisitorische Informationen, Rechte und Finanzmittel in die Betrachtung mit einbezogen, wie sie in Abbildung 1 graphisch dargestellt sind.

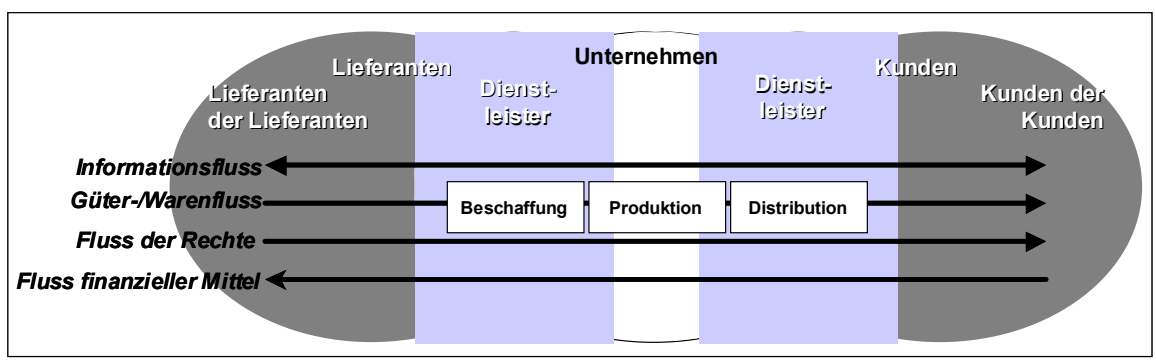

Abbildung 1: Flussbetrachtung im Supply Chain Management [Pfoh00].

Das über Unternehmensgrenzen hinweg flussorientierte Supply Chain Management erfordert in besonderem Maße die Berücksichtigung von Schnittstellen sowie ein hohes $\mathrm{Ma} \beta$ an interner und externer Kooperation. Im Mittelpunkt stehen Kosten-, Zeit- und Qualitätsziele der gesamten Supply Chain gemessen am Nutzen für den Endkunden.

\subsection{Akteure und Sichtweisen im Financial Supply Chain Management-Verständnis}

Die Aufgaben, Akteure und Sichtweisen im Financial Supply Chain Management wurden im Rahmen eines Arbeitskreises der Bundesvereinigung Logistik (BVL) gemeinsam mit dem Fachgebiet Unternehmensführung \& Logistik der Technischen Universität Darmstadt erarbeitet [Pfoh +03$]$. Ausgangspunkt der Financial Supply Chain ist die Logistik mit ihren Schnittstellen und Interdependenzen zu Beschaffung/Einkauf, Produktion sowie Distribution/Marketing. Zusammen mit den Funktionen Finanzierung, Investition 
und Rechnungswesen sind sie Gegenstand der funktionalen Sichtweise (Abbildung 2). Dabei zählen erstere zum „klassischen“ Supply Chain-Verständnis und letztere zum „erweiterten“ Verständnis einer Financial Supply Chain .

Handlungsträger in der Financial Supply Chain sind neben Lieferanten, logistischen Dienstleistern und Kunden insbesondere die Institutionen, die Finanzierungs- und Investitionsleistungen anbieten. Diese können sowohl innerhalb des eigenen Unternehmens (z.B. Finanzabteilung), als auch außerhalb (z.B. Banken, Finanzdienstleister und Investoren), angeordnet sein. Die Betrachtung der Akteure in der Financial Supply Chain führt zur institutionalen Sichtweise. In dieser lassen sich die Finanz- und Logistikwelt innerhalb des Unternehmens (mikroinstitutional) und über Unternehmensgrenzen hinweg (makroinstitutional) definieren und voneinander abgrenzen.

Die finanzwirtschaftliche Sichtweise lässt sich in einen strom- und bestandsgrößenorientierten Teil aufspalten. Hier spiegeln sich die güterwirtschaftlichen Leistungsprozesse einer Supply Chain im Finanzmittelfluss wider. Im klassischen Supply Chain-Verständnis fallen unter die finanzwirtschaftliche Sichtweise vor allem Prozesskosten, Deckungsbeiträge sowie das Anlage- und Umlaufvermögen. Diese bilden im Wesentlichen die Bestands- und Stromgrößen des Leistungsbereichs ab. Durch den Einbezug von Investition und Finanzierung - im funktionalen sowie institutionalen Sinne dehnt sich die Betrachtung im erweiterten Supply Chain-Verständnis auf Bestands- und Stromgrößen wie beispielsweise Kapitalkosten, Cash Flows und risikoadäquate Renditen aus [Pfoh+03]. Das erweiterte Verständnis einer Financial Supply Chain ist in Abbildung 2 zusammenfassend dargestellt.

\begin{tabular}{|c|c|c|c|c|}
\hline \multirow{2}{*}{$\begin{array}{l}\text { Sichtweisen } \\
\text { Elemente ... }\end{array}$} & \multirow[t]{2}{*}{ Funktional } & \multicolumn{2}{|c|}{ Institutional } & \multirow{2}{*}{$\begin{array}{l}\text { Finanzwirtschaftlich } \\
\text { (Bestands-\& Stromgrößen) }\end{array}$} \\
\hline & & Makro-Akteure & Mikro-Akteure & \\
\hline $\begin{array}{l}\text {... im } \\
\text { klassischen } \\
\text { Supply Chain } \\
\text { Verständnis }\end{array}$ & $\begin{array}{l}\text { - Beschaffung/ } \\
\text { Einkauf } \\
\text { - Produktion } \\
\text { - Distribution/ } \\
\text { Marketing } \\
\text { - Logistik }\end{array}$ & $\begin{array}{l}\text { - Logistik- } \\
\text { dienstleister } \\
\text { - Industrie } \\
\text { - Handel } \\
\text { - Kunden } \\
\text { - Lieferanten }\end{array}$ & $\begin{array}{l}\text { Abteilungen } \\
\text { und Akteure } \\
\text { des Leistungs- } \\
\text { bereichs } \\
\text { (z.B. Logistik- } \\
\text { abteilung) }\end{array}$ & $\begin{array}{l}\text { - Prozesskosten } \\
\text { - Deckungsbeiträge } \\
\text { - Anlage- und } \\
\text { Umlaufvermögen } \\
\text { der Logistik }\end{array}$ \\
\hline $\begin{array}{l}\ldots \text { aus Sicht der } \\
\text { Financial Supply } \\
\text { Chain }\end{array}$ & $\begin{array}{l}\text { - Rechnungswesen/ } \\
\text { Controlling } \\
\text { - Finanzierung } \\
\text { - Investition }\end{array}$ & $\begin{array}{l}\text { - Investoren } \\
\text { - Finanz- } \\
\text { dienstleister } \\
\text { (z.B. Banken, } \\
\text { Rating- } \\
\text { Agenturen) }\end{array}$ & $\begin{array}{l}\text { Finanz- und } \\
\text { Rechnungs- } \\
\text { wesen- } \\
\text { abteilung }\end{array}$ & $\begin{array}{l}\text { - Kapitalkosten } \\
\text { - Cash Flow } \\
\text { - risikoadäquate } \\
\text { Rendite }\end{array}$ \\
\hline
\end{tabular}

Abbildung 2: Klassisches und erweitertes Verständnis der Supply Chain [Pfoh+03]. 


\subsection{Die Financial Chain in der Supply Chain}

Für viele Primärprozesse wurden Fortschritte in der IuK-Technologie zur Automatisierung, zur internen und externen Vernetzung und letztlich zur Neuaufteilung der Wertschöpfungskette genutzt. Dagegen sind die Finanzflüsse bislang in den wenigsten Fällen auf eine Prozessoptimierung ausgerichtet und immer noch stark durch die Aufbauorganisation bestimmt. Wird in Betracht gezogen, dass die Finanzflüsse im Durchschnitt ein Viertel der gesamten ITBudgets benötigen, lohnt ein Blick auf potenzielle Verbesserungsmöglichkeiten in diesem Bereich [Skie+03].

Die Aktivitäten in der Financial Chain fallen in den meisten Unternehmen unter die unterstützenden Sekundärprozesse, die im Gegensatz zu den primären Leistungserstellungsprozessen der Supply Chain, keinen direkten Beitrag zur Wertschöpfung der Unternehmen liefern. Sie liegen dadurch viel zu selten im Blickpunkt des Top-Managements [Skie+03].

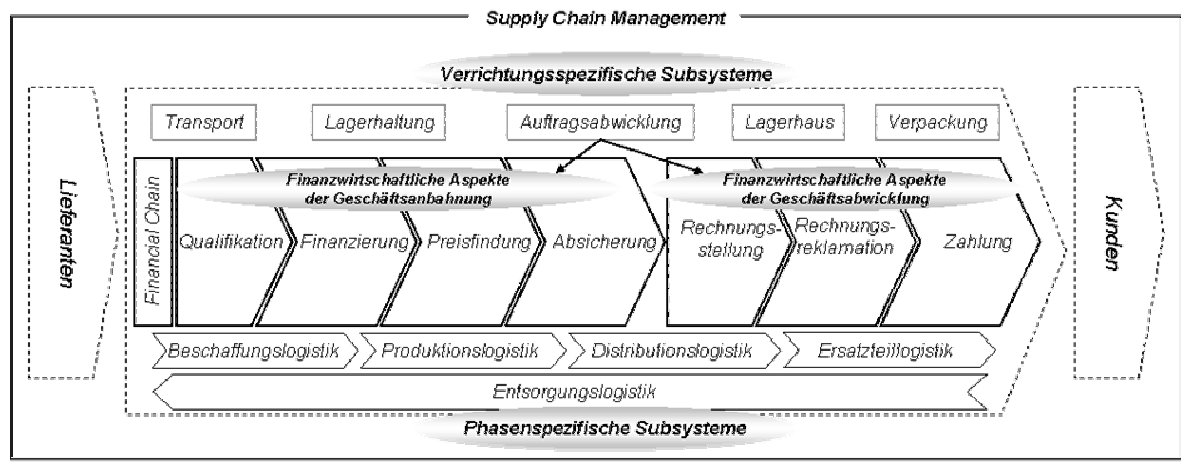

Abbildung 3: Generische Financial Chain eingebettet in das Supply Chain Management

Die aus sieben Teilschritten bestehende Financial Chain wird als intra- und interorganisationaler Prozess in der Supply Chain betrachtet [Pfaf +03$]$. Die Geschäftsanbahnung beginnt mit der Qualifikation der Geschäftspartner, indem Identität und Bonität des Kunden überprüft werden. Danach wird die Finanzierung des Geschäftes geregelt, etwa durch einen Lieferantenkredit oder Leasing. Es folgen Preisverhandlung und -festsetzung (Preisfindung) und anschließend die Angebotserstellung sowie die Absicherung von Währungs-, Transport- und Kreditausfallrisiken. Die eigentliche Erfüllung des Geschäftes erfolgt durch die oben beschriebenen phasen- und verrichtungsspezifischen Subsysteme der Logistik, die im Rahmen des Supply Chain Managements mit Lieferanten und Kunden synchronisiert werden. Nach dem Erfüllungsgeschäft beginnt die Geschäftsabwicklung mit der Rechnungsstellung und deren Versand sowie eventuelle Rechnungsreklamationen durch den Kunden. Sind eventuelle 
Beanstandungen der Rechnung beseitigt, erfolgt die Zahlung der Rechnung durch den Kunden. Die Financial Chain betont damit den finanziellen Aspekt der Auftragsabwicklung und lässt sich als Teilprozess in das erweiterte Supply Chain Management-Verständnis integrieren. Wie u.a. [Weit04] zeigen, ist diese generische Financial Chain in nahezu allen deutschen Unternehmen in dieser Form vorhanden.

\section{Wertsteigerung durch Financial Chain Management}

Um die Financial Chain zu steuern und deren Effizienz auf Unternehmensebene zu messen, sind Kennzahlen nötig. Als kapitalmarkttheoretisch konsistentes und auf Grund der guten Anwendbarkeit in der Praxis weit verbreitetes Wertsteigerungsmaß hat sich die Discounted Cash Flow-Methode erwiesen [Cope+00; NiSt03]. Im Folgenden wird zunächst die Discounted Cash FlowMethode als Wertsteigerungsmaß für das Management der Financial Chain erläutert. Darauf aufbauend werden die Wertsteigerungsmaße für zwei Prozessschritte aus der generischen Financial Chain exemplarisch dargestellt und empirisch analysiert.

\subsection{Wertsteigerungsmaß für die Financial Chain}

Die Maximierung des eigenen Unternehmenswertes stellt für jeden Akteur der Supply Chain ein grundlegendes Ziel dar. Zur Ermittlung des Unternehmenswertes exisitieren in Theorie und Praxis eine Reihe unterschiedlicher Ansätze und Methoden. Diese Ansätze und Methoden lassen sich unterscheiden in traditionelle Verfahren (z.B. substanzorienterte Bewertungsverfahren und Ertragswertverfahren) und in kapitalmarktorientierte Verfahren (z.B. Discounted Cash Flow (DCF), Shareholder Value Ansatz, Economic Value Added (EVA) und Cash Flow Return on Investment (CFRoI)). Siehe dazu die Übersichten bei [Pape99; Rapp99].

Im folgenden Abschnitt wird der Discounted Cash Flow als Wertsteigerungsmaß des Unternehmenswertes für das Financial Chain Management eingeführt.

\section{Discounted Cash Flow-Methode}

Der Shareholder Value berechnet sich entsprechend der Discounted Cash FlowMethode aus der Differenz von Unternehmenswert und Marktwert des Fremdkapitals [Rapp99]. Für die Berechnung des Unternehmenswertes ist der Barwert der Free Cash Flows während der Prognoseperiode auf Basis der Cash Flows zu berechnen [Cope+00]. Auf diese Weise können Interdependenzen von 
Selbstfinanzierung und Investitionen sowie die Auswirkungen von Investitionen auf die Liquidität des Unternehmens aufgezeigt werden. Zusätzlich wird der jährliche Zuwachs an zur Verfügung stehenden finanziellen Mitteln deutlich. Dabei findet folgendes Berechnungsschema Anwendung [Sieg94]:

Cash Flow

- Steuerausgaben

- Anlageinvestitionen

- Dividenden

- Zunahme Nettoumlaufvermögen

\section{$=$ Free Cash Flow}

Der Free Cash Flow lässt sich als Zielgröße für die Analyse, Ausarbeitung und Beurteilung von Alternativen im Rahmen des Financial Chain Managements zu Grunde legen. Eine Bedingung dafür stellt bei einer wertorientierten Unternehmensführung die Identifikation von Werttreibern dar. Diese können mit Hilfe der Berechnungsverfahren des DCF systematisch aufgedeckt und auf ihren Einfluss zur Wertsteigerung hin untersucht werden.

\section{Shareholder Value-Netzwerk}

Zur Ermittlung der Einflussgrößen des DCF kann das Shareholder ValueNetzwerk [Rapp99] herangezogen werden. Es verdeutlicht die wichtigsten Interdependenzen zwischen dem Unternehmenswert als Zielgröße, und den finanziellen Werttreibern des DCF, wie sie in Abbildung 4 dargestellt sind [Knor98]. Die dunkel hinterlegten Werttreiber des Shareholder Value-Netzwerkes können durch Financial Chain Management direkt beeinflusst werden. Der Einfluss wird im Folgenden exemplarisch an zwei Prozessschritten verdeutlicht. 


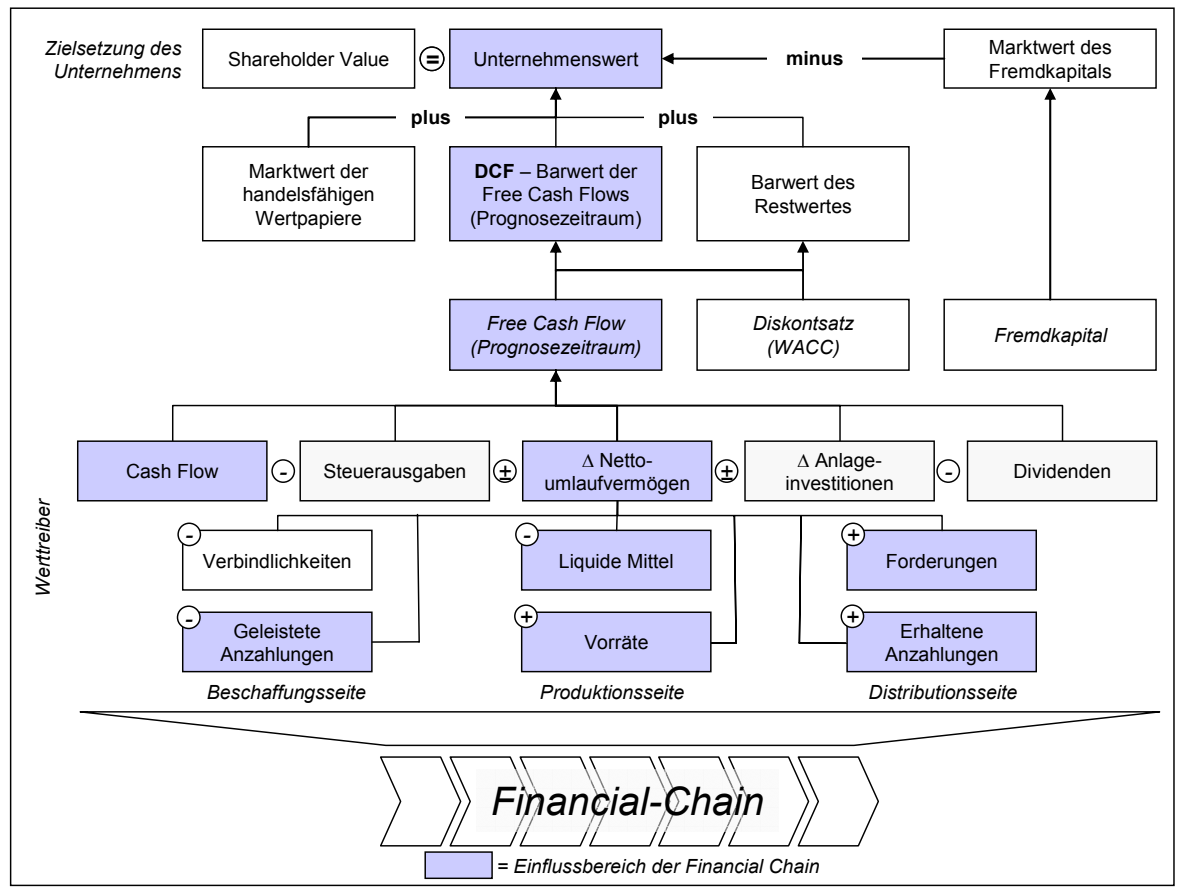

Abbildung 4: Shareholder Value-Netzwerk von Rappaport [Rapp99] ergänzt um eine Werttreiberhierarchie bei indirekter Berechnung der Free Cash Flows aus Verkäufersicht.

\subsection{Werttreiber für das Financial Chain Management}

Von den sieben Teilprozessen der Financial Chain werden den Teilprozessen Rechnungsstellung (26\%) und Qualifikation (19\%) in den Teilbereichen Geschäftsanbahnung und -abwicklung jeweils die höchsten Verbesserungspotenziale zugeordnet [Skie+03]. Deren Einflussmöglichkeiten auf den Shareholder Value werden im Folgenden analysiert.

\section{Qualifikation}

Der Teilprozessschritt Qualifikation ist der Geschäftsanbahnung zuzuordnen. Eine schnellere Qualifikation wirkt sich durch einen geringeren Lagerbestand positiv auf die Lagerhaltungskosten aus. Dies kann durch eine schnellere Authentifizierung und Bonitätsprüfung, die bei Unternehmen im Durchschnitt etwa 27 Tage dauert, erreicht werden, da dem Kunden das Produkt schneller zugestellt und somit die Vorräte und Lagerhaltung reduziert werden können [Arkh02a; Pfaf+04a]. Eine Steigerung der Qualität der Qualifikation, z.B. durch 
die Einbindung von externen Daten (z.B. Dun \& Bradstreet) oder die Integration von internen Daten (z.B. vergangenenes Zahlungsverhalten) kann eine Verbesserung des Forderungsportfolios und eine Reduzierung des Ausfallrisikos bewirken. Dadurch wird der Forderungsbestand verbessert [Pfoh +03 ; ZiMe02]. Eine verbesserte Qualifikation und das korrekte Einschätzen eines Zahlungsausfallrisikos kann zudem die Anzahlungen erhöhen. So sollte beispielswiese bei einem Kunden, bei dem das Zahlungsausfallrisiko hoch ist, entweder eine Absicherung in Form einer Versicherung [Pfaf $+04 \mathrm{a}$ ] oder eine Anzahlung verlangt werden. Die Ausführungen zum Prozessschritt der Qualifikation verdeutlichen dessen Relevanz für den Unternehmenswert. So kann der Free Cash Flow durch eine richtige Einschätzung der Kunden und der Einleitung präventiver Maßnahmen verbessert werden.

\section{Rechnungsstellung}

Der Teilprozessschritt Rechnungsstellung ist ein Teil der Geschäftsabwicklung und wirkt unmittelbar auf die Forderungen und liquiden Mittel. Der Cash Flow kann hier durch Kosteneinsparungen positiv beeinflusst werden. Diese Kosteneinsparungen können entweder durch die Veränderung des Ausgangskanals, z.B. durch den verstärkten Einsatz elektronischer Rechnungen [Hasc02; Stef02; Trad01], oder die Realisierung von Skaleneffekten [Blum04], z.B. durch Shared Service Center, erzielt werden [Kage01; CaRu01]. Auch der Forderungsbestand kann durch eine Beschleunigung des Rechnungsstellungsprozesses reduziert werden. So kann der elektronische Rechnungsversand die Außenstandstage (Days Sales Outstanding - DSO) reduzieren und somit den Kapitalrückfluss in das Unternehmen beschleunigen [Hasc02]. Aber auch eine Reduzierung der Fehlerquote bei der Rechnungsstellung und Fakturabearbeitung kann den DSO reduzieren [SkPf03]. Eine Reduzierung der Außenstandstage hat auch eine Erhöhung der liquiden Mittel und somit eine Verbesserung des Working Capital zur Folge.

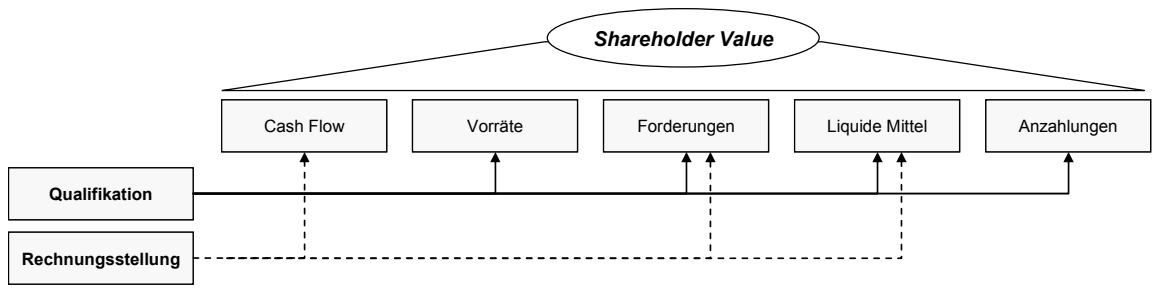

Abbildung 5: Wirkungen der Prozessschritte Qualifikation und Rechnungsstellung auf die Werttreiber des DCF

Durch geeignete Verbesserungen des Teilprozessschrittes Rechnungsstellung kann eine positive Wirkung auf den Cash Flow und den Forderungsbestand und somit auf die liquiden Mittel eines Unternehmens erzielt werden. Der Unternehmenswert lässt sich demnach auch durch eine Verbesserung (z.B. Beschleunigung, 
Reduzierung der Fehlerquote) des Rechnungsstellungsprozesses steigern. In Abbildung 5 sind die identifizierten Wirkungen der beiden untersuchten Prozessschritte Qualifikation und Rechnungsstellung auf die Werttreiber des Shareholder Value-Netzwerkes dargestellt.

\section{Empirische Analyse der Financial Chain}

Die diesem Artikel zugrunde liegende Studie zu Verbesserungspotenzialen in der Financial Chain unter den größten deutschen Unternehmen wurde 2003 am Lehrstuhl für Betriebswirtschaftslehre, insbesondere Electronic Commerce und dem Institut für Wirtschaftsinformatik der J. W. Goethe-Universität in Frankfurt als Projekt des E-Finance Labs durchgeführt. Dazu wurde ein Fragebogen mit 35 teils weiter detaillierten Fragestellungen auf acht Seiten mit offenen und geschlossenen Fragen an die Finanzverantwortlichen der umsatzstärksten 1.000 Unternehmen (ohne Finanzdienstleister) in Deutschland geschickt [Skie+03]. Der Fragebogen gliederte sich in Fragestellungen zu den sieben Teilprozessen sowie übergreifenden Fragestellungen zur Financial Chain. Im Vorlauf der Studie wurden alle Unternehmen telefonisch kontaktiert, um die Finanzverantwortlichen zu identifizieren. Diesen wurde dann der Fragebogen direkt zugeschickt. Der Fragebogen wurde in Pre-Tests und Expertengesprächen auf Verständlichkeit und inhaltliche Konsistenz geprüft und angepasst. Die Studie war dabei auf die Branchen ausgerichtet, in denen die Prozesse der Financial Chain keine Primäroder Kernprozesse darstellen, d.h. Banken und sonstige Finanzdienstleister wurden ausgeschlossen. In einer schriftlichen und einer telefonischen Nachfassaktion wurden alle Finanzverantwortlichen erneut kontaktiert. Die Rücklaufquote der Befragung lag bei 10,3\%, dies entspricht einer Gesamtzahl von 103 Datensätzen.

\subsection{Optimierungspotenzial in der Financial Chain}

In diesem Abschnitt werden die Prozessoptimierungspotenziale der einzelnen Teilprozesse der Financial Chain untersucht. Es werden die Einbindung des Teilprozesses in den Gesamtablauf der Financial Chain sowie teilprozessspezifische Schwachstellen aufgezeigt.

Neben dem hohen Optimierungspotenzial in den Teilprozessen Qualifikation und Rechnungsstellung sehen die befragten Finanzverantwortlichen weitere Verbesserungsmöglichkeiten in den Teilprozessen Rechnungsreklamation (19\%), Preisfindung (16\%), Zahlung (14\%), Finanzierung (13\%) und Absicherung (10\%). Durch Mehrfachnennung summieren sich die einzelnen Anteile auf über $100 \%$. Den finanzwirtschaftlichen Aspekten der Geschäftsanabwicklung wird im 
Vergleich zu der Geschäftsanbahnung im Mittel ein tendenziell höheres Optimierungspotenzial zugesprochen.

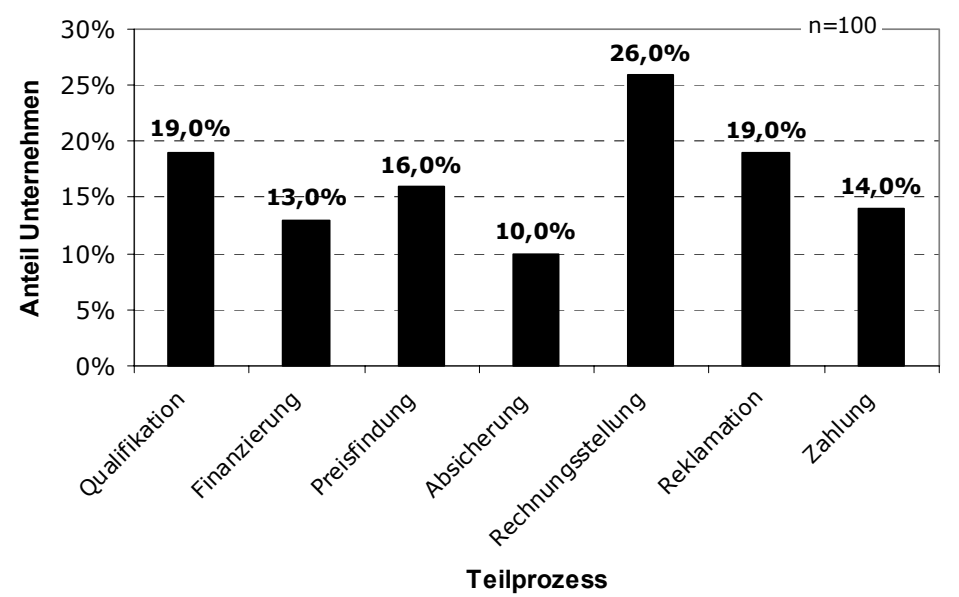

Abbildung 6: Optimierungspotenzial der Financial Chain-Teilprozesse

Im Rahmen der empirischen Befragung wurden sowohl Schwachstellen in den Teilprozessen als auch die relevanten Einflussfaktoren (Reduktion der Prozessdauer, Automatisierungsgrad, Optimierung der IT, Optimierung der Abläufe, bessere Schnittstellen $\mathrm{zu}$ anderen Teilprozessen, Outsourcing, standardisierte Abläufe) erhoben. Bei der Analyse der Schwachstellen und Optimierungsfaktoren zeigt sich für den Bereich der Anbahnung eines Geschäftsprozesses aus finanzwirtschaftlicher Sicht, dass eine Optimierung der Abläufe des Teilprozesses stets als ein wichtiger oder sogar der wichtigste Faktor zur Erschließung von Verbesserungspotenzial erachtet wird. Für den Teilprozess der Qualifikation ist kein spezifischer Schwerpunkt feststellbar, alle Faktoren werden als etwa gleichbedeutend erachtet. In den Teilprozessen Finanzierung und Preisfindung erhoffen sich die Befragten Einsparpotenziale insbesondere durch die Optimierung der Prozessabläufe und die Reduktion der Prozessdauer der Finanzflüsse. Im Teilprozess Absicherung spielt schließlich eine bessere Ausgestaltung der Schnittstellen zwischen den Teilprozessen eine herausragende Rolle.

In der Abwicklung werden im Teilprozess Rechnungsstellung nahezu alle Faktoren als hochgradig relevant für eine weitere Optimierung des Teilprozesses angesehen. Es heben sich hierbei insbesondere die Optimierung des Prozessablaufs und die Ausgestaltung besserer Schnittstellen $\mathrm{zu}$ anderen Teilprozessen als besonders wichtige Faktoren mit jeweils 80\% positiver Nennungen hervor. Weiterhin wird die Reduktion der Prozessdauer der Finanzflüsse als sehr wichtig eingestuft. 
Eine Reduktion der Prozessdauer verspricht für viele Unternehmen im Teilprozess Rechnungsreklamation vorteilhaft zu sein (89,5\%). Auch hier wird erneut eine Optimierung der Prozessabläufe als zentraler Optimierungsfaktor angesehen (78,9\%). Sowohl die allgemeine Optimierung der Abläufe als auch die Reduzierung der Durchlaufzeit bieten nach Einschätzung der Unternehmen für den Teilprozess Rechnungsreklamation nachhaltiges Optimierungspotenzial. Eine Optimierung der IT-Systeme (38,9\%) oder die Auslagerung an einen externen Partner $(22,2 \%)$ scheinen dagegen hier eine eher untergeordnete Rolle zu spielen. $\mathrm{Da}$ es sich bei Rechnungsreklamationen in hohem Maße um einen nicht standardisierten Prozess handeln dürfte (durch die große Anzahl möglicher Rechnungsreklamationsgründe), erscheint eine Automatisierung des Prozesses und eine damit verbundene Optimierung der Informationstechnologie nicht angebracht zu sein. Durch die hohe Individualisierung der einzelnen Rechnungsreklamationsanfragen ist hier das Potenzial einer Optimierung der Informationstechnologie aus Sicht der Unternehmen eher gering.

Im letzten Teilprozess der Financial Chain, der Zahlung, sind es erneut die Optimierung der Prozessabläufe wie auch die Reduktion der Prozessdauer, die von der überwiegenden Anzahl der befragten Unternehmen als vorteilhaft angesehen werden $(84,7 \%)$. Auch die Optimierung der IT-Systeme scheint in diesem Bereich, der im Gegensatz etwa zum Teilprozess Rechnungsreklamation einen hohen Grad standardisierter Abläufe aufweisen kann, zur weiteren Optimierung des Teilprozesses gut geeignet zu sein $(83,3 \%)$.

\subsection{Wirkung der Teilprozesse auf den Werttreiber Forderungen}

Die empirischen Ergebnisse zeigen, dass eine Reduktion bei der Prozessdauer neben der Optimierung der Prozessabläufe als wichtigster Faktor innerhalb der einzelnen Teilprozessschritte der Financial Chain gesehen wird. Der Zeitraum zwischen der Stellung einer Rechnung und der dazugehörigen Zahlung, also das Kundenzahlungsverhalten, kann durch die Kennzahl DSO operationalisiert werden [Pfaf $+04 b ;$ Stan03]. Geringe DSO reduzieren den Forderungsbestand und erhöhen damit die liquiden Mittel im Unternehmen. Sie reduzieren die Dauer bis das Kapitel in das Unternehmen zurückfliesst. Im Folgenden soll daher exemplarisch die Wirkung der Teilprozesse auf den Werttreiber Forderungen dargestellt werden.

In der Vergangenheit wurden die Zielverkäufe und die daraus resultierenden Außenstände von Zahlungen und Forderungen auf ineffiziente Mahn- und Geldeinzugsprozeduren zurückgeführt. Heute wird die Financial Chain zunehmend in Bezug auf die DSO betrachtet, um so die Gründe von Außenständen zu hinterfragen. Je länger die Kunden, begründet oder unbegründet, die Zahlung hinauszögern, desto länger ist der gesamte Cash Cycle eines Unternehmens [Seil99; Stan03]. Der Cash Cycle beschreibt dabei die Kapitalbindung eines Unternehmens. Er entspricht dem Zeitraum von der 
Bezahlung der Rohstoffe und Materialien durch das Unternehmen und der Bezahlung der Lieferungen und Leistungen durch die Kunden.

Bei der Betrachtung der DSO ist zu beachten, dass das eigentliche Zahlungsziel einen erheblichen Einfluss hat. Wenn beispielsweise ein Unternehmen seinen Kunden ein sehr langes Zahlungsziel gewährt, so werden auch die DSO sehr hoch sein. Dies wird in der Studie durch eine signifikant starke Korrelation nach Pearson $(0,855)$ zwischen Zahlungsziel und DSO bestätigt. Daher wurde die Netto-DSO als Differenz zwischen DSO und Zahlungsziel gebildet. Die NettoDSO hat unter den Top-1.000-Unternehmen einen Mittelwert von 8, d. h. Kunden zahlen im Durchschnitt 8 Tage später als das eigentlich vereinbarte Zahlungsziel. Die Spannweite der Netto-DSO liegt zwischen -22 und 76 Tagen. Die negativen Zahlen der Netto-DSO zeigen, dass einige Kunden ihre Rechungen vor dem eigentlichen Zahlungsziel begleichen.

Es wurde nun untersucht, welche Faktoren einen Einfluss auf die DSO haben. Zu diesen Faktoren zählen interne Informationen wie beispielsweise das Zahlungsverhalten in der Vergangenheit, externe Informationen wie beispielsweise eine Bonitätsprüfung durch die Schufa, der verwendete Rechnungsausgangskanal sowie die Zahlungsmethode. Die Ergebnisse zeigen, dass die DSO umso geringer sind, je mehr interne Informationen in die Zahlungskonditionen mit einfließen (hoch signifikante Korrelation, $\mathrm{p}<=0,001$, nach Pearson: 0,302). Bei der Überprüfung, ob dieselben Zusammenhänge auch für externe Informationen gelten, wurden keine signifikanten Zusammenhänge festgestellt. Die empirische Analyse zeigt ebenfalls, dass die Anzahl der unterschiedlichen Zahlungskonditionen einen Einfluss auf die DSO haben. Je weniger unterschiedliche Zahlungskonditionen vorliegen, desto geringer sind die DSO (schwach signifikante Korrelation, $\mathrm{p}<0,10$, nach Pearson: -0,202).

Auch die anderen Prozessschritte wurden in die Analyse mit einbezogen. So konnte kein signifikanter Zusammenhang zwischen dem Kanal des Rechnungsversandes und den DSO festgestellt werden. Aussagen, dass elektronische Rechungen die DSO reduzieren, können somit anhand dieser Studie nicht bestätigt werden. Andere Studien zeigen allerdings, dass ein elektronischer Versand der Rechnungen durchaus die DSO reduzieren kann [Pfaf +04$]$.

Im Gegensatz zum Kanal des Rechnungsversands hat die Zahlungsmethode nachweisbaren Einfluss auf die DSO. So ist eine positive Korrelation zwischen den DSO und der Zahlungsmethode „Überweisung“ festzustellen (hoch signifikante Korrelation nach Pearson: 0,385). Ebenfalls eine positive Korrelation ist zwischen den DSO und der Scheckzahlung erkennbar (signifikante Korrelation, $\mathrm{p}<=0,05$, nach Pearson: 0,197). Beide Zahlungsmethoden sind tendenziell mit höheren DSO und damit einem späteren Zahlungseingang verbunden. Die Ergebnisse verwundern nicht, da es sich hierbei um Zahlungsmethoden handelt, die vom Zahlungspflichtigen, also dem Kunden ausgelöst werden. Im Gegensatz dazu ist das Lastschriftverfahren zu sehen. Dort wird die Zahlung durch den 
Zahlungsempfänger eingeleitet. Auch hier wurde eine signifikante Korrelation festgestellt, die, wie erwartet, negativ ist (hoch signifikante Korrelation nach Pearson: -0,379).

Um die Erkenntnisse der Ergebnisse $\mathrm{zu}$ validieren, wurde ebenfalls die Korrelation zwischen kompletten Zahlungen und den Netto-DSO überprüft. Die Erwartung, dass komplette Zahlungen von Rechnungen die DSO reduzieren, konnte anhand der Stichprobe gezeigt werden (signifikante Korrelation nach Pearson: -0,207). Ebenfalls kann mit den empirischen Erkenntnissen gezeigt werden, dass Rechnungen, die nicht bezahlt werden, die DSO erhöhen (signifikante Korrelation nach Pearson: 0,307). Die DSO werden ebenfalls durch zu Recht bemängelte Rechnungen der Kunden verlängert (hoch signifikante Korrelation nach Pearson: 0,311).

\subsection{Handlungsoptionen für Banken}

Die Gestaltung der Financial Chain ist nicht Teil der Kernkompetenz der meisten Industrie-, Handels- und Dienstleistungsunternehmen. Damit stellt sich die Frage, inwiefern Banken und Finanzdienstleister ihre Kompetenz in der Gestaltung von Finanzprozessen den Unternehmen (z.B. als Sourcingpartner) zur Verfügung stellen können, um die Financial Chain zu optimieren [Pfoh +03$]$. Weiterhin stellen Banken bereits jetzt integrale Teile der Financial Supply Chain dar, da die Abwicklung der Zahlung selbst und die Reallokation der Zahlungsmittel praktisch stets über das Bankennetzwerk erfolgt. Zudem zählen die Kreditvergabe und die Entwicklung von Absicherungsinstrumenten für Unternehmen zu den von Banken und Finanzdienstleistern innerhalb der Supply Chain bereitgestellten Dienstleistungen.

Den Banken wird in den Teilprozessen Finanzierung und Absicherung das größte Potenzial zugesprochen [Skie+03]. Weitere Studien sehen ebenfalls eine zukünftige Rolle der Banken in der Finanzierung, der Absicherung, der Rechnungsstellung und dem Teilprozess der Zahlung [Arkh02b; Fair03; Pfaf $+04 c$ ] [Pfoh +03$]$.

Outsourcing spielt in der Einschätzung der Unternehmen im Bereich der Geschäftsanbahnung der Financial Chain keine herausragende Rolle. Auch im Prozessschritt der Rechnungsstellung wird, wie schon bei den Prozessen der Geschäftsanbahnung, das Outsourcing des Teilprozesses an einen externen Dienstleister nur von wenigen Unternehmen $(16,7 \%)$ in Betracht gezogen.

Sollten Finanzdienstleister, insbesondere Banken, sich dazu entschliessen ein kompetenter Partner für die Übernahme von Prozessen in der Financial Chain zu werden, oder als Sourcingpartner neue Erlösquellen erschließen wollen, ist eine frühe aktive Rolle der Banken und Finanzdienstleister in der Kundenbeziehung eine notwendige Voraussetzung [Skie +03 ]. 
Das Potenzial für Banken und Finanzdienstleister, Dienstleistungen innerhalb der Financial Chain zu übernehmen, wird heute zwar nur von einem geringen Teil der befragten Unternehmen gesehen. Da jedoch nur wenige Unternehmen explizit mit ihre Financial Chain zufrieden sind, können sich hier durchaus neue Geschäftsfelder für Banken und Finanzdienstleister ergeben [Pfaf $+04 c]$.

\section{Handlungsempfehlungen und Fazit}

Die Betrachtung der Finanzflüsse wurde im Supply Chain Management-Konzept bislang vernachlässigt, da die Geschäftsanbahnung und -abwicklung häufig nicht den Kernprozessen der traditionellen Akteure einer Supply Chain zugerechnet wird. Erst in jüngster Zeit stehen die Finanzflüsse der Supply Chain - das Financial Supply Chain Management - im Mittelpunkt der wissenschaftlichen Diskussion. Die Identifikation der Werttreiber ist dabei eine notwendige Voraussetzung zur Realisation von Prozessverbesserungen.

Wie eine empirische Untersuchung der Financial Chain der Top-1.000 Unternehmen zeigt, weist die Reduktion der Prozessdauer der Teilprozesse das größte Potenzial auf. Dabei spielt sowohl die interne als auch die externe Integration eine zentrale Rolle. Die Analyse der DSO zeigt, dass einzelne Maßnahmen durchaus den Cash Flow Cycle reduzieren und so gebundenes Kapital der Unternehmen freisetzen können. Dieses Kapital kann anderweitig eingesetzt werden und so den Unternehmenswert erhöhen. In diesem Beitrag wurden exemplarisch die Qualifikation als finanzwirtschaftlicher Teilprozess der Geschäftsanbahnung und die Rechnungsstellung als finanzwirtschaftlicher Teilprozess der Geschäftsabwicklung bezüglich ihre Wirkung auf den Free Cash Flow untersucht, um Verbesserungspotenziale in der Financial Chain zu ermitteln. Gerade in diesem Punkt wird deutlich, wie wichtig neue und innovative Ansätze zum Cash Management und Treasury sind. Auch die elektronische Rechnungsstellung kann ihren Beitrag zu einer Reduzierung des Working Capital leisten. Hier entstehen neue Geschäftspotenziale für die Finanzdienstleistungsbranche. Diese Potenziale sind durch eine umfassende elektronische Unterstützung bzw. eine Optimierung bestehender Systeme der Financial Chain in und zwischen Unternehmen zu realisieren. Erste IT-Lösungen finden sich beispielsweise in der SAP Solution Map „Financial Supply Chain Management" oder in den Produkten von TradeCard [Pfaf $+04 a]$.

Die Entwicklung von Werttreibern für zwei Teilprozessschritte in diesem Beitrag deutet an, welche Potenziale im Financial Chain Management realisiert werden können. Zukünftige Forschungsaufgaben umfassen eine detaillierte Analyse der Werttreiber der hier nicht betrachteten Teilprozessschritte der Financial Chain, eine Analyse der Beschaffungsprozesse und eine Ausweitung auf die gesamte Wertschöpfungskette. 


\section{Literatur}

[Arkh02a] Arkhipov, A. V.: Benchmarking Financial Operations Processes and Technologies. Report, Aberdeen Group Inc.: Boston, 2002.

[Arkh02b] Arkhipov, A. V.: Best Practices in Streamlining the Financial Value Chain. Report, Aberdeen Group Inc.: Boston, 2002.

[Ball+00] Ballou, R. H.; Gilbert, S. M.; Mukherjee, A.: New Managerial Challenges from Supply Chain Opportunities. Industrial Marketing Management 29, 2000, S. 7-18.

[Blum04] Blumenberg, S.: Benchmarking Financial Chain Efficiency - the Role of Economies of Scale for Financial Processes. Proceedings of the Eighth PacificAsia Conference on Information System (PACIS), Shanghai, 2004.

[Coop+97] Cooper, M. C.; Lambert, D. M.; Pagh, J. D.: Supply Chain Management: More than a New Name for Logistics. The International Journal of Logistics Management 8, 1997, S. 1-14.

[Cope+00] Copeland, T.; Koller, T.; Murrin, J.: Valuation: Measuring and Managing the Value of Companies. John Wiley \& Sons: New York et al., 2000.

[CLM04] Council of Logistics Management (CLM): Supply Chain Management/Logistics Management Definitions. http://clm1.org/Website/AboutCLM/Definitions/ Definitions.asp, 2004, Abruf am 29.06.2004.

[Fair03] Fairchild, A. M.: Value Positions for Financial Institutions in Electronic Bill Presentment and Payment (EBPP). Proceedings of the 36th Hawaii International Conference on System Sciences, Hawaii, 2003.

[Harr95] Harrington, L.: Logistics, Agent for Chance: Shaping the Integrated Supply Chain. Journal of Transportation \& Distribution 36, 1995, S. 30-34.

[Hasc02] Haschka, J. M.: Will EIPP Ever Kill the Wholesale Lockbox Business? AFP Exchange 22, 2002, o.S.

[Kage01] Kagelmann, U.: Shared Services als alternative Organisationsform - Am Beispiel der Finazfunktion im multinationalen Konzern. Gabler Edition Wissenschaft: Wiesbaden, 2001.

[KePf04] Keller, G.; Pfaff, D.: Wertorientierte Geschäftsprozessgestaltung in heterogenen Systemlandschaften am Beispiel einer deutschen Retailbank. Banking Information Technology (BIT) 2004, S. 23-34.

[Kill02] Killen \& Associates Inc.: Optimizing The Financial Supply Chain - How CFO of Global Enterprises Are Succeding by Substituting Information for Working Capital. Arbeitsbericht: Palo Alto, Californien, 2002.

[Knor98] Knorren, N.: Wertorientierte Gestaltung der Unternehmensführung. Wiesbaden, 1998.

[LaCo00] Lambert, D. M.; Cooper, M. C.: Issues in Supply Chain Management. Industrial Marketing Management 29, 2000, S. 65-83. 
[Lamb+98] Lambert, D. M.; Cooper, M. C.; Pagh, J. D.: Supply Chain Management: Implementation Issues and Research Opportunities. The International Journal of Logistics Management 9, 1998, S. 1-19.

[LaHa04] Larson, P. D.; Halldorsson, A.: Logistics Versus Supply Chain Management. An International Survey. International Journal of Logistics: Research and Applications 7, 2004, S. 17-31.

[NiSt03] Nippel, P.; Streitferdt, F.: Unternehmensbewertung mit dem WACC-Verfahren: Steuern, Wachstum und Teilausschüttung. Schmalenbachs Zeitschrift für betriebswirtschaftliche Forschung 55, 2003, S. 401-422.

[Pape99] Pape, U.: Wertorientierte Unternehmensführung und Controlling. Berlin, 1999.

[Pfaf+04] Pfaff, D.; Keller, G.; Skiera, B.: Prozessverbesserungen durch eine Automatisierung der Finanzströme am Beispiel der Rechnungsstellung. Working Paper, Frankfurt, 2004.

[Pfaf+04a] Pfaff, D.; Skiera, B.; Weiss, J.: Financial Supply Chain Management. GalileoVerlag: Bonn, 2004.

[Pfaf+03] Pfaff, D.; Skiera, B.; Weitzel, T.: Datenintegration in Finanzprozessen. Informatik 2003 - Innovative Informatikanwendungen; Beiträge der 33. Jahrestagung der Gesellschaft für Informatik e. V.; GI-Edition, Frankfurt, 2003, S. 165-168.

[Pfaf $+04 b]$ Pfaff, D.; Skiera, B.; Weitzel, T.: Financial-Chain-Management: Ein generisches Modell zur Identifizierung von Verbesserungspotenzialen. Wirtschaftsinformatik 46, 2004, S. 107-117.

$[$ Pfaf $+04 c]$ Pfaff, D.; Skiera, B.; Wrobel, M.; Stockinger, W.: Geschäftspotenziale für Banken in der industriellen Financial Chain. Die Bank 2004, 1, S. $62-65$.

[Pfoh00] Pfohl, H.-C.: Supply Chain Management: Logistik Plus? Logistikkette. Marketingkette. Finanzkette. Erich Schmidt Verlag: Berlin, 2000.

[Pfoh04] Pfohl, H.-C.: Logistiksysteme. Betriebswirtschaftliche Grundlagen. Berlin/Heidelberg, 2004.

[Pfoh+03] Pfohl, H.-C.; Elbert, R.; Hofmann, E.: Management der "finanziellen" SupplyChain: Charakterisierung - Aufgabenbereiche - Interdependenzen. In: (BVL), B. L. (Hrsg): Finanzierung - eine neue Dimension der Logistik. Berlin, 2003, S. 164.

[Rapp99] Rappaport, A.: Shareholder Value. Ein Handbuch für Manager und Investoren. Stuttgart, 1999.

[Seil99] Seiler, A.: Financial Management - BWL in der Praxis II. Zürich, 1999.

[Sieg94] Siegwart, H.: Der Cash-flow als finanz- und ertragswirtschaftliche Lenkungsgröße. Stuttgart, 1994.

[Skie+03] Skiera, B.; König, W.; Gensler, S.; Weitzel, T.; Beimborn, D.; Blumenberg, S.; Franke, J.; Pfaff, D.: Financial-Chain-Management, Prozessanalyse, Effizienzpotentiale und Outsourcing-Eine Empirische Studie mit den 1.000 
Größten Deutschen Unternehmen. Arbeitsbericht: E-Finance Lab: Frankfurt, 2003.

[SkPf03] Skiera, B.; Pfaff, D.: Financial Supply Chain Management - Wie Sie Ihren Cash Cycle in den Griff bekommen! Der Controlling Berater 2003, S. 47-68.

[Stan03] Stanley, J.: DSO Resolution Network. The Complete Solution. Credit Management 2003, S. 40-42.

[Stef02] Stefanidis, C.: Why Hasn't Electronic Bill Presentment and Payment Taken Off? Federal Reserve Bank of New York: Current Issues in Economics and Finance 8, 2002 ,

[Trad01] TradeCard: Financial Supply Chain Automation: The Missing Link in Supply Chain Management. White Paper, New York, http:// www.tradecard.com/resources/newsArticles/WorldTrade_090100.pdf, 2001.

[CaRu01] von Campenhausen, C.; Rudolf, A.: Shared Services-profitabel für vernetzte Unternehmen. Harvard Business Manager 23, 2001, S. 82-93.

[EiJü03] von Eisenhart-Rothe, F.; Jütte, S.: Innovative Logistikfinanzierung rund um die Supply Chain. In: Bundesvereinigung Logistik (BVL), A. F. u. L. (Hrsg): Finanzierung - eine neue Dimension der Logistik. Berlin, 2003, S. 151-170.

[Weit04] Weitzel, T.; Beimborn, D.; Franke, J.: Outsourcing the Financial Chain: an Empirical Analysis of Sourcing and Partnering Potentials. Tenth American Conference on Information Systems (AMCIS), New York, 2004.

[ZiMe02] Zipfel, C.; Meindl, H.: Unternehmensliquidität im Griff. Financial Supply Chain Management. Controller Magazin 27, 2002, S. 555-557. 